\title{
FAMILIES OF NEGATIVELY CURVED HERMITLAN MANIFOLDS
}

\author{
MICHAEL J. COWEN
}

Abstract. A complex analytic family of compact hermitian manifolds has negative holomorphic sectional curvature in a neighborhood of any fibre having negative holomorphic sectional curvature.

1. Introduction. In [1, Hilfssatz 4, p. 120], Grauert and Reckziegel state:

If $(Y, \pi, X)$ is an analytic family of compact Riemann surfaces of genus $\geqq 2$, over a Riemann surface $X$, then for each point $x_{0}$ in $X$ there is a neighborhood $V \subset X$ of $x_{0}$ and a differential metric on $Y \mid V$ such that $Y \mid V$ is strongly negatively curved.

Their construction of the metric is clear, but the proof of strong negative curvature involves a computation which is not altogether complete. (The proof, however, can be completed easily using the formula for the Gaussian curvature of the sum of two hermitian metrics [1, Aussage 1, p. 111].) The purpose of this note is to give a simpler computation which shows that the metric actually has holomorphic sectional curvature $\leqq c<0$ and hence a fortiori is strongly negatively curved [2, p. 39]. Indeed we will show that if the fibres of $Y$ are $n$-dimensional compact manifolds each having holomorphic sectional curvative less than a negative constant, then $Y \mid V$ has negative holomorphic sectional curvature.

2. Definitions and statement of results. Let $d s^{2}$ be a hermitian metric on an $n$-dimensional complex manifold $M$, with $d s^{2}=\sum g_{i j} d z_{i} d \bar{z}_{j}$ in local coordinates. Define the curvature tensor by

$$
K_{i j k m}=\frac{\partial^{2} g_{i j}}{\partial z_{k} \partial \bar{z}_{m}}-\sum_{p, q} \frac{\partial g_{i p}}{\partial z_{k}} g^{p q} \frac{\partial g_{q j}}{\partial \bar{z}_{m}} \text { for } 1 \leqq i, j, k, m \leqq n .
$$

$M$ has holomorphic sectional curvature (which will be denoted by h.s.c.) less than a constant $c$ if $-\sum k_{i j k m} s_{i} \bar{s}_{j} s_{k} \bar{s}_{m}<c$ for all holomorphic unit tangent vectors $s=\sum s_{i} \partial / \partial z_{i}$.

Received by the editors June 5, 1972.

AMS (MOS) subject classifications (1970). Primary 53C55; Secondary 32G05, 32H20.

Key words and phrases. Holomorphic sectional curvature, hyperbolic manifold, analytic family, differential metric.

(c) American Mathematical Society 1973 
THEOREM. Let $(Y, \pi, X)$ be an analytic family of compact complex $n$-dimensional manifolds over a Riemann surface $X$, such that for each $x_{0} \in X$, the fibre $Y_{x_{0}}=\pi^{-1}\left(x_{0}\right)$ has a hermitian metric of h.s.c. $<c<0$, then there exists a neighborhood $V$ of $x_{0}$ in $X$ such that $Y \mid V$ has a hermitian metric of h.s.c. $<c^{\prime}<0$, with $c, c^{\prime}$ constants.

REMARK. This generalizes Grauert and Reckziegel's result since a compact Riemann surface of genus $\geqq 2$ has a hermitian metric of Gaussian curvature (equals h.s.c. on a Riemann surface) less than a negative constant [2, Theorem 5.1, p. 12]

COROLlaRY. If $\sigma$ is a holomorphic section of $Y$ with isolated singularities in $X$, then $\sigma$ extends as a holomorphic section to all of $X$.

3. Construction of the metric. The construction is the obvious generalization of that in [1].

Since $(Y, \pi, X)$ is locally trivial we can find a neighborhood $V$ with coordinate $z_{n+1}$ centered at $x_{0}$, and neighborhoods $U_{1}, \cdots, U_{r}$ in $Y$ such that $Y \mid V=\bigcup U_{m}$, each $U_{m}$ has coordinates $z_{1}, \cdots, z_{n+1}$ with

$$
\pi\left(z_{1}, \cdots, z_{n+1}\right)=z_{n+1},
$$

and $z_{1}, \cdots, z_{n}$ give coordinates in $U_{m} \cap Y_{z_{n+1}}$ for all $z_{n+1}$ in $V$. The hermitian metric on $Y_{0}=Y_{x_{0}}$ is of the form $\sum g_{i j}\left(z_{1}, \cdots, z_{n}\right) d z_{i} d \bar{z}_{j}$ on $U_{m} \cap Y_{0}$ and thus can be extended to a pseudo-hermitian metric $\sum h_{i j} d z_{i} d \bar{z}_{j}$ on $U_{m}, 1 \leqq i, j \leqq n$, by setting $h_{i j}\left(z_{1}, \cdots, z_{n+1}\right)=g_{i j}\left(z_{1}, \cdots, z_{n}\right)$. These pseudo-hermitian metrics can then be patched together by a partition of unity to give a pseudo-hermitian metric $\alpha$ on $Y \mid V$, such that $\alpha \mid Y_{0}$ is the original hermitian metric on $Y_{0}$. That is, $\alpha=\sum k_{i j} d z_{i} d \bar{z}_{j}(1 \leqq i, j \leqq n+1)$ on $U_{m}$ and $k_{i j}\left(z_{1}, \cdots, z_{n}, 0\right)=g_{i j}\left(z_{1}, \cdots, z_{n}\right)$ for $1 \leqq i, j \leqq n$. Since $\alpha \mid Y_{0}$ has h.s.c. $<c<0$, it is clear that for $z_{n+1}$ close enough to $0, \alpha \mid Y_{z_{n+1}}$ will have h.s.c. $<c<0$. By shrinking $V$ we can assume (1) $V=\left\{\left|z_{n+1}\right|<t\right\}$, (2) $\alpha \mid Y_{z_{n+1}}$ has h.s.c. $<c<0$ for all $z_{n+1} \in V$, and (3) $k_{i j}$, its first, and second partial derivatives are bounded on each $U_{m}$ for $1 \leqq i, j \leqq n+1$. Since a disc in $C$ has a hermitian metric of Gaussian curvature - 1, we can put $V$ in a larger disc and obtain a metric $h\left(z_{n+1}\right) d z_{n+1} d \bar{z}_{n+1}$ on $V$ of Gaussian curvature $=-1$ such that $h$, its first, and second partials are bounded on $V$. Define a metric $d s^{2}$ on $Y \mid V$ for each $\lambda>0$ by $d s^{2}=\alpha+\lambda h\left(z_{n+1}\right) d z_{n+1} d \bar{z}_{n+1}$, i.e. on $U_{m}, d s^{2}=\sum k_{i j} d z_{i} d \bar{z}_{j}+\lambda h\left(z_{n+1}\right) d z_{n+1} d \bar{z}_{n+1}$. Note that for large $\lambda$, $d s^{2}$ has negative h.s.c. in both the fibre and base directions. We wish to choose $\lambda_{0}$ so that for all $\lambda \geqq \lambda_{0}, d s^{2}$ will have h.s.c. $\leqq c^{\prime \prime}<0$. Clearly it suffices to do this on each $U_{m}$ and then take the maximum of the $\lambda_{0}$ 's so obtained. 
4. Proof of negative sectional curvature. Assume we have shown the following:

(i) $K_{i j k m} \rightarrow \tilde{K}_{i j k m}$ for $1 \leqq i, j, k, m \leqq n$, uniformly on $U_{m}$ as $\lambda \rightarrow \infty$, where $\tilde{K}_{i j k m}\left(z_{1}, \cdots, z_{n+1}\right)$ is the curvature of $d s^{2}$ restricted to $Y_{z_{n+1}} \cap U_{m}$.

(ii) $\left|K_{i j k m}\right| \leqq M$ on $U_{m}$ for all $1 \leqq i, j, k, m \leqq n+1$ except when $i=j=$ $k=m=n+1$, and $M$ is a constant.

$$
K_{i j k m}=\lambda\left(\frac{\partial^{2} h}{\partial z_{n+1} \partial \bar{z}_{n+1}}-\frac{1}{h} \frac{\partial h}{\partial z_{n+1}} \frac{\partial h}{\partial \bar{z}_{n+1}}\right)+O(1),
$$

when $i=j=k=m=n+1$, where $O(1)$ means a term which is uniformly bounded on $U_{m}$.

Since the Gaussian curvature of $h$ is

$$
\frac{1}{h}\left(\frac{\partial^{2} h}{\partial z_{n+1} \partial \bar{z}_{n+1}}-\frac{1}{h} \frac{\partial h}{\partial z_{n+1}} \frac{\partial h}{\partial \bar{z}_{n+1}}\right) \leqq-1
$$

and $h$ is bounded on $U_{m}$, we have:

(iii) $K_{i j k m} \leqq \lambda c^{\prime}$ when $i=j=k=m=n+1$, where $c^{\prime}<0$ is a constant, for $\lambda \geqq \lambda_{0}$.

Fix $z=\left(z_{1}, \cdots, z_{n+1}\right)$. If $s=\sum_{i=1}^{n} s_{i}\left(\partial / \partial z_{i}\right)$ is a holomorphic unit tangent vector to the fibre $Y_{z_{n+1}}$ then by (i) we have

$$
-\sum K_{i j k m} s_{i} \bar{s}_{j} s_{k} \bar{s}_{m} \rightarrow-\sum \tilde{K}_{i j k m} s_{i} \bar{s}_{j} s_{k} \bar{s}_{m}<c<0 \text { as } \lambda \rightarrow \infty .
$$

Hence by compactness of the unit sphere, we can choose $\lambda_{0}$ large enough so that for $\lambda \geqq \lambda_{0}$ we have $-\sum K_{i j k m} s_{i} \bar{s}_{j} s_{k} \bar{s}_{m}<c$ for $s$ tangent to the fibre. But if $s=\sum_{i=1}^{n+1} s_{i} \partial / \partial z_{i}$ is any holomorphic unit tangent vector, then by (ii) and (iii)' we have:

$$
\begin{aligned}
-\sum K_{i j k m} s_{i} \bar{s}_{j} s_{k} \bar{s}_{m} \leqq & -\sum_{i, j, k, m=1}^{n} K_{i j k m} s_{i} \bar{s}_{j} s_{k} \bar{s}_{m} \\
& +M \sum^{\prime}\left|s_{i}\right|\left|s_{j}\right|\left|s_{k}\right|\left|s_{m}\right|+\lambda c^{\prime}\left|s_{n+1}\right|^{4},
\end{aligned}
$$

where $\Sigma^{\prime}$ is the sum of the terms where at least one, but not all, of the $i, j, k, m$ equals $n+1$. Thus if $s$ is not tangent to the fibre, i.e., $s_{n+1} \neq 0$, then by taking $\lambda_{0}$ large enough we can insure that the h.s.c. is less than $c_{s}$ in a neighborhood of $s$ on the unit sphere, for all $\lambda \geqq \lambda_{0}$. But from (*) it is also clear that if $s$ is tangent to the fibre, then the h.s.c. is less than $c_{s}$ in a neighborhood of $s$ for all $\lambda \geqq \lambda_{0}$. Therefore for each fixed $z$ the h.s.c. at $z$ is less than $c_{z}$ for $\lambda>\lambda_{0}$ and hence by the relative compactness of $U_{m}$, the h.s.c. $<c<0$ on $U_{m}$ for $\lambda \geqq \lambda_{0}$, which proves the Theorem.

Let $d s^{2} \mid Y_{z_{n+1}}=\sum \tilde{k}_{i j} d z_{i} d \bar{z}_{j}$ be the metric restricted to the fibre, where $\tilde{k}_{i j}=k_{i j}$ for $1 \leqq i, j \leqq n$. Since

$$
d s^{2}=\sum k_{i j} d z_{i} d \bar{z}_{j}+\lambda h\left(z_{n+1}\right) d z_{n+1} d \bar{z}_{n+1} \equiv \sum g_{i j} d z_{i} d \bar{z}_{j}
$$


where $1 \leqq i, j \leqq n+1$, it is easy to check that:

(a) $g^{i j}=\tilde{k}^{i j}+O\left(\lambda^{-1}\right), \quad \partial g_{i p} / \partial z_{k}=\partial \tilde{k}_{i p} / \partial z_{k}, \quad \partial^{2} g_{i j} / \partial z_{k} \partial \bar{z}_{m}=\partial^{2} \tilde{k}_{i j} / \partial z_{k} \partial \bar{z}_{m}$ for $1 \leqq i, j, k, m, p \leqq n$.

$$
\begin{aligned}
g^{i j} & =\lambda^{-1} h\left(z_{n+1}\right)^{-1}+O\left(\lambda^{-2}\right), \quad \frac{\partial g_{i p}}{\partial z_{n+1}}=O(1)+\frac{\partial h}{\partial z_{n+1}}, \\
\frac{\partial^{2} g_{i j}}{\partial z_{n+1} \partial \bar{z}_{n+1}} & =O(1)+\lambda \frac{\partial^{2} h}{\partial z_{n+1} \partial \bar{z}_{n+1}} \quad \text { for } i=j=p=n+1 .
\end{aligned}
$$

(c) $g^{i j}=O\left(\lambda^{-1}\right), \partial g_{i p} / \partial z_{k}=O(1), \partial^{2} g_{i j} / \partial z_{k} \partial \bar{z}_{m}=O(1)$ otherwise. (Note. Since $h$ is a function only of $z_{n+1}$, terms such as $\partial g_{i p} / \partial z_{k}$, for $i=p=n+1$ but $k \neq n+1$, do not involve $\lambda$ or the derivatives of $h$.)

If $1 \leqq i, j, k, m \leqq n$ then

$$
\begin{aligned}
K_{i j k m} & =\frac{\partial^{2} \tilde{k}_{i j}}{\partial z_{k} \partial \bar{z}_{m}}-\sum_{p, q=1}^{n} \frac{\partial \tilde{k}_{i p}}{\partial z_{k}}\left(\tilde{k}^{p q}+O\left(\lambda^{-1}\right)\right) \frac{\partial \tilde{k}_{q j}}{\partial \bar{z}_{k}}+O\left(\lambda^{-1}\right) \\
& =\widetilde{K}_{i j k m}+O\left(\lambda^{-1}\right),
\end{aligned}
$$

which proves (i). If $i=j=k=m=n+1$, then

$$
\begin{aligned}
K_{i j k m}= & \lambda \frac{\partial^{2} h}{\partial z_{n+1} \partial \bar{z}_{n+1}}+O(1)-\sum_{p, q=1}^{n} \frac{\partial g_{i p}}{\partial z_{k}} O\left(\lambda^{-1}\right) \frac{\partial g_{q j}}{\partial \bar{z}_{m}} \\
& -\sum_{p=1}^{n} \frac{\partial g_{i p}}{\partial z_{k}} O\left(\lambda^{-1}\right) O(1)-\sum_{q=1}^{n} O(1) O\left(\lambda^{-1}\right) \frac{\partial g_{q j}}{\partial \bar{z}_{k}} \\
& -\left(O(1)+\lambda \partial h / \partial z_{n+1}\right)\left(\lambda^{-1} h^{-1}+O\left(\lambda^{-2}\right)\right)\left(O(1)+\lambda\left(\partial h / \partial \bar{z}_{n+1}\right)\right) \\
= & \lambda\left(\partial^{2} h / \partial z_{n+1} \partial \bar{z}_{n+1}\right)-h^{-1}\left(\partial h / \partial z_{n+1}\right)\left(\partial h / \partial \bar{z}_{n+1}\right) \\
& +O(1)+O\left(\lambda^{-1}\right)+O\left(\lambda^{-2}\right)
\end{aligned}
$$

which proves (iii). The proof of (ii) is obvious, since the only terms which are not $O(1)$ or $O\left(\lambda^{-1}\right)$ are those appearing only when $i=j=k=m=n+1$.

5. Proof of Corollary. Assume $\sigma$ has an isolated singularity at $x_{0} \in H$. By the Theorem, there is a neighborhood $V=\{|z|<1\}$ of $x_{0}$ such that $Y \mid V$ has a metric of h.s.c. $<c<0$. Thus by [2, Theorem 4.11, p. 61], $Y \mid V$ is hyperbolic and, by a theorem of Mrs. Kwack [2, Theorem 3.1, p. 83], $\sigma: V-\{0\} \rightarrow Y \mid V$ has a holomorphic extension to $\sigma^{\prime}: V \rightarrow Y \mid V$ if there exists a suitable sequence of points $x_{n} \rightarrow x_{0}$ such that $\sigma\left(x_{n}\right) \rightarrow p_{0} \in$ $Y \mid V$. Since $Y \mid V$ is relatively compact in $Y$, the result follows.

6. Remarks. That $X$ is a Riemann surface was not crucial to the proof of the Theorem and the proof goes through with obvious modifications when $X$ is an arbitrary complex manifold. Then in the Corollary, $\sigma$ 
need only have singularities contained in an analytic set of codimension $\geqq 1$ in $X$, for $\sigma$ to extend to all of $X$. The proof of the Corollary then follows from a result of Mrs. Kwack [2, Theorem 4.1, p. 86].

\section{REFERENCES}

1. H. Grauert and H. Reckziegel, Hermitesche Metriken und normale Familien holomorpher Abbildungen, Math. Z. 89 (1965), 108-125. MR 33 \#2827.

2. S. Kobayashi, Hyperbolic manifolds and holomorphic mappings, Pure and Appl. Math., no. 2, Marcel Dekker, New York, 1970. MR 43 \#3503.

Department of Mathematics, Tulane University, New Orleans, Louisiana 70118

Current address: Department of Mathematics, Princeton University, Princeton, New Jersey 08540 